CrossMark

\title{
Home-based maintenance tele- rehabilitation reduces the risk for acute exacerbations of COPD, hospitalisations and emergency department visits
}

\author{
Maroula Vasilopoulou ${ }^{1,2}$, Andriana I. Papaioannou ${ }^{3}$, Georgios Kaltsakas ${ }^{2}$, \\ Zafeiris Louvaris ${ }^{2}$, Nikolaos Chynkiamis ${ }^{1,2}$, Stavroula Spetsioti ${ }^{2}$, \\ Eleni Kortianou², Sofia Antiopi Genimata², Anastasios Palamidas², \\ Konstantinos Kostikas ${ }^{4}$, Nikolaos G. Koulouris ${ }^{2}$ and loannis Vogiatzis ${ }^{1,2,5}$
}

Affiliations: ${ }^{1}$ Faculty of Physical Education and Sport Sciences, National and Kapodistrian University of Athens, Athens, Greece. ${ }^{2}$ First Dept of Respiratory Medicine, National and Kapodistrian University of Athens, Sotiria General Chest Hospital, Athens, Greece. ${ }^{3}$ Third Respiratory Medicine Dept, Sismanogleion General Hospital, Athens, Greece. ${ }^{4}$ Second Dept of Respiratory Medicine, National and Kapodistrian University of Athens, Attikon General Hospital, Athens, Greece. ${ }^{5}$ Faculty of Health and Life Sciences, Dept of Sport, Exercise and Rehabilitation, Northumbria University Newcastle, Newcastle Upon Tyne, UK.

Correspondence: loannis Vogiatzis, Faculty of Health and Life Sciences, Dept of Sport, Exercise and Rehabilitation, Northumbria University Newcastle, Newcastle Upon Tyne, NE1 8ST, UK.

E-mail: ioannis.vogiatzisanorthumbria.ac.uk

@ERSpublications

Home tele-rehabilitation reduces risk of COPD exacerbation; is effective alternative to in-hospital rehabilitation http://ow.ly/T17g30ap9cY

Cite this article as: Vasilopoulou M, Papaioannou AI, Kaltsakas G, et al. Home-based maintenance telerehabilitation reduces the risk for acute exacerbations of COPD, hospitalisations and emergency department visits. Eur Respir J 2017; 49: 1602129 [https://doi.org/10.1183/13993003.02129-2016].

ABSTRACT Pulmonary rehabilitation (PR) remains grossly underutilised by suitable patients worldwide. We investigated whether home-based maintenance tele-rehabilitation will be as effective as hospital-based maintenance rehabilitation and superior to usual care in reducing the risk for acute chronic obstructive pulmonary disease (COPD) exacerbations, hospitalisations and emergency department (ED) visits.

Following completion of an initial 2-month PR programme this prospective, randomised controlled trial (between December 2013 and July 2015) compared 12 months of home-based maintenance telerehabilitation $(n=47)$ with 12 months of hospital-based, outpatient, maintenance rehabilitation $(n=50)$ and also to 12 months of usual care treatment $(n=50)$ without initial PR.

In a multivariate analysis during the 12-month follow-up, both home-based tele-rehabilitation and hospital-based PR remained independent predictors of a lower risk for 1) acute COPD exacerbation (incidence rate ratio (IRR) $0.517,95 \%$ CI $0.389-0.687$, and IRR 0.635 , 95\% CI 0.473-0.853), respectively, and 2) hospitalisations for acute COPD exacerbation (IRR 0.189 , 95\% CI $0.100-0.358$, and IRR 0.375 , 95\% CI 0.207-0.681), respectively. However, only home-based maintenance tele-rehabilitation and not hospital-based, outpatient, maintenance PR was an independent predictor of ED visits (IRR 0.116, 95\% CI 0.072-0.185).

Home-based maintenance tele-rehabilitation is equally effective as hospital-based, outpatient, maintenance PR in reducing the risk for acute COPD exacerbation and hospitalisations. In addition, it encounters a lower risk for ED visits, thereby constituting a potentially effective alternative strategy to hospital-based, outpatient, maintenance PR.

This article has supplementary material available from erj.ersjournals.com

Received: Oct 312016 | Accepted after revision: Feb 162017

Copyright OERS 2017 


\section{Introduction}

The benefits of pulmonary rehabilitation (PR) in terms of improvement in functional capacity, limb muscle function, chronic dyspnoea and emotional function are well documented [1]. However, these benefits are often lost over the subsequent 12 months without the implementation of a maintenance strategy $[1,2]$. Few investigations have explored maintenance strategies to sustain the benefits of PR over the longer term; these results remain equivocal in regard to the duration that functional capacity and quality of life are preserved [3-6]. The effect of these long-term strategies to reduce the risk for acute exacerbations of chronic obstructive pulmonary disease (COPD), hospitalisations and emergency department visits (ED) also remains inconclusive $[3,4,6,7]$. A recent meta-analysis of 10 randomised controlled trials (including both short- and long-term PR maintenance strategies) [7] demonstrated that $\mathrm{PR}$, when compared with usual care, is associated with lower overall rates of hospitalisations attributable to acute exacerbations of COPD.

Despite the well-documented benefits of PR [1], long-term access and utilisation of PR by eligible COPD patients remains low due to insufficient funding, resources, reimbursement and other patient-related barriers limiting long-term access to PR [8]. Accordingly, alternative approaches (such as tele-rehabilitation) to extend the initial benefits of PR for a longer term were recently recommended by the official American Thoracic Society (ATS)/European Respiratory Society (ERS) policy statement [8].

Tele-rehabilitation involves the use of information and communication technologies to provide rehabilitation services remotely to people in their homes [9]. In contrast to traditional centre-based programmes, undertaking PR within the home environment might promote more effective, longer-term integration of exercise routines into daily life [10]. Although there is still little evidence of the benefits of tele-rehabilitation [1], initial small-scale studies, most of which were uncontrolled, demonstrate some feasibility. These data suggest that clinical benefits such as compliance, patient empowerment to physical training, improved health status and quality of life might be achieved [10-14]. Although these look promising, these interventions had numerous limitations such as a short duration intervention (2-6 months), high dropout rates (up to $45 \%)$, small patient numbers $(n \leqslant 10)$ or poor adherence to different components of tele-rehabilitation [10-13, 15-17].

Consequently, more evidence is needed to ascertain the effectiveness of home-based maintenance tele-rehabilitation in reducing the risk for acute exacerbations of COPD, hospitalisations and ED visits, while maintaining the functional benefits of primary PR. In the present prospective study we hypothesised that regular home monitoring of vital signs, in combination with tele-consultation sessions encouraging patient adherence to physical training regimens, would be as effective as hospital-based, outpatient, maintenance rehabilitation and superior to usual care in reducing the risk for acute exacerbations of COPD, hospitalisations and ED visits over a 12 -month period.

\section{Methods and materials Study design}

As shown in figure 1, 150 COPD patients were randomised into three groups using a set of computer-generated random numbers to either home-based maintenance tele-rehabilitation (group A, n=50), hospital-based, outpatient, maintenance rehabilitation (group $\mathrm{B}, \mathrm{n}=50$ ) or usual care treatment (group $\mathrm{C}, \mathrm{n}=50$ ), (ClinicalTrials. gov identifier: NCT02618746). During the period spanning from December 2013 to July 2015, patients in groups A and B initially completed a multidisciplinary intense hospital-based, outpatient, PR programme lasting for 2 months (supplementary material [18]), which was followed by a 12-month maintenance rehabilitation programme at home (group A) or at hospital (group B). Patients in group C followed the usual care treatment throughout the 14-month period, without participation to either the 2-month primary or the 12-month maintenance programmes (figure 1). In Greece, only few university medical departments deliver PR. Hence, the majority of COPD patients follow usual care only, which is associated with high costs for treating acute exacerbations of COPD. The present study was designed to investigate the value of delivering and maintaining the benefits of initial PR compared with usual care. Thus, to balance the observation period between the three groups, we performed all measurements in group $\mathrm{C}$ at the same time points as in the other two groups (i.e. months 0,2 and 14).

Support statement: This work (TELECARE: 11SYN_10_1438) was co-financed by Greece (General Secretariat for Research and Technology) and the European Union via the National Strategic Reference Framework (NSRF 2007-2013; Competitiveness and Entrepreneurship). Funding information for this article has been deposited with the Crossref Funder Registry.

This study is registered at the clinicaltrials.gov online database with identifier NCT02618746.

Conflict of interest: Disclosures can be found alongside this article at erj.ersjournals.com 


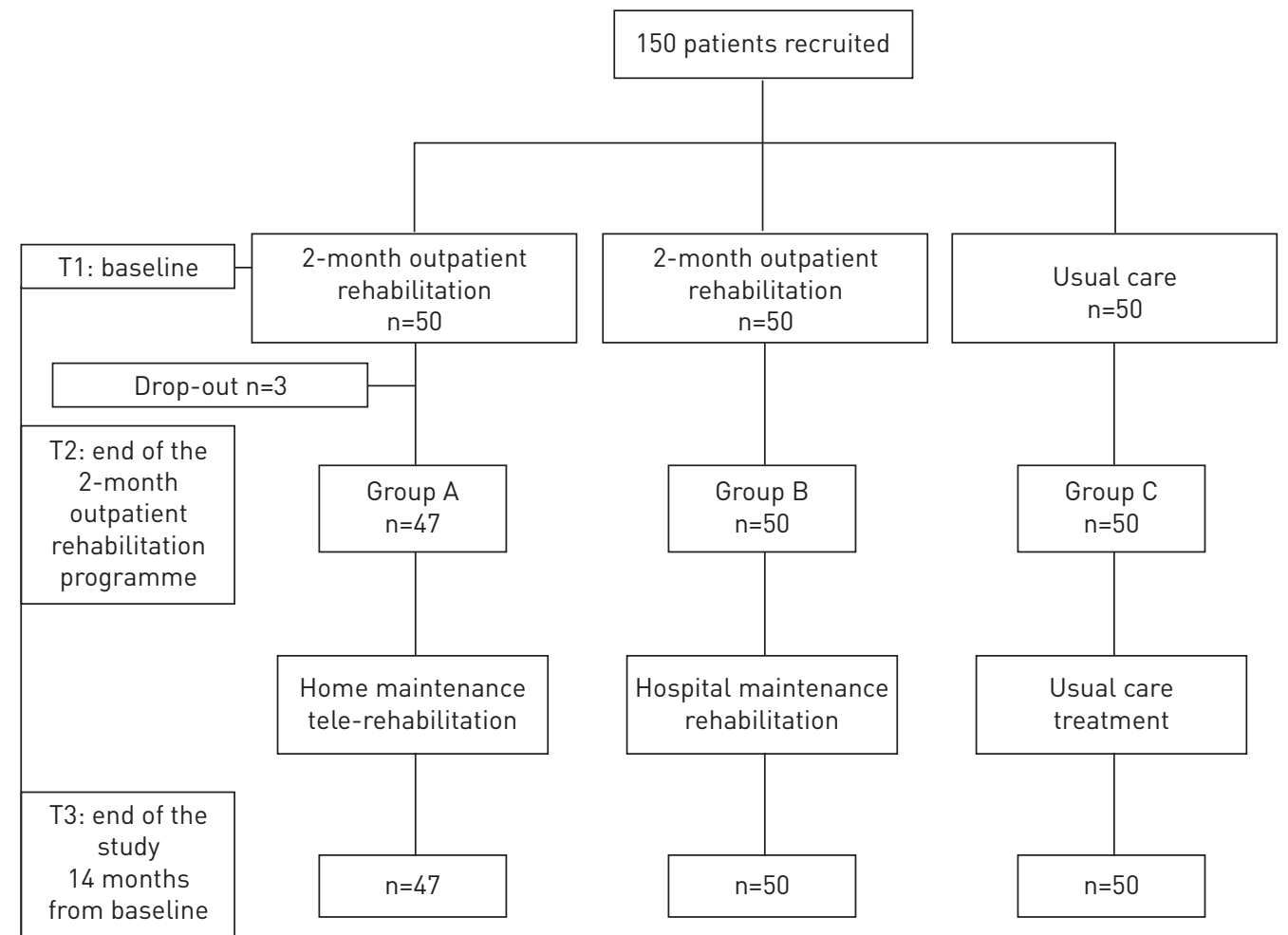

FIGURE 1 Patient flow chart providing information in regards to the study protocol.

\section{Study subjects}

150 clinically stable patients that regularly attending the Outpatient Clinic (1st Dept of Respiratory Medicine at Athens University Medical School based at Sotiria General Chest Hospital, Athens, Greece) were recruited. Patients $(n=150)$ were included in the study if they were older than 40 years; with a diagnosis of COPD (post-bronchodilation forced expiratory volume in $1 \mathrm{~s}$ (FEV1)/forced vital capacity $($ FVC) $<0.7$ ) with moderate to very severe airflow obstruction (post-bronchodilator FEV $1<80 \%$ pred); with optimal medical treatment according to the Global Initiative for Chronic Obstructive Lung Disease (GOLD) [19] without regular use of systemic corticosteroids; and a history of acute exacerbations of COPD 1 year prior to entering the study. Patients were excluded from the study based on the diagnosis of orthopaedic, neurological and other conditions that significantly impair exercise tolerance, or respiratory disorders other than COPD. Patients were also excluded on grounds of cognitive impairment and/or difficulties to managing electronic devices that precluded interactions with the tablet, as judged by the investigator. More details on the exclusion criteria are provided in the supplementary material. The majority of patients were referred to the PR programme because of persistent respiratory symptoms, but also following hospitalisation for acute exacerbation of COPD (four patients in group A and six patients in group B). These patients were included in the study at least 8 weeks after the hospitalisation [19]. None of these patients had previously participated in a PR programme. The Scientific Board of Clinical Studies at Sotiria Hospital approved the study protocol (approval number: 22964).

\section{Description of interventions}

\section{Home-based maintenance tele-rehabilitation programme (group A)}

The home-based maintenance tele-rehabilitation programme consisted of 144 sessions performed over 12 months. The programme included the following components: 1) individualised action plan; 2) physical exercise sessions to remote monitoring; 3) access to the call centre 5 days per week, $10 \mathrm{~h}$ per day; 4) psychological support; and 5) dietary and self-management advice via scheduled weekly contacts with a physiotherapist, an exercise scientist, a dietician and a physician through telephone or a video conference. During the course of the 2-month initial PR programme, patients were trained to appropriately use the multimodal apparatus (MIR Spirodoc, Spirodoc ${ }^{\circ}$, Spiro+Oxi, Rome, Italy) that was subsequently used to make measurements at home. Training enabled the patients to take their own spirometry and vital sign measurements using a wireless apparatus fitted with Bluetooth technology. Patients were also taught to successfully transfer the data to a tablet placed in their home (Lenovo Smart Tab II ${ }^{7}$, Bratislava, Slovakia) and transmit the data from the tablet to a secured web-based platform via a mobile communications 
network. The home-based exercise programme comprised arm and leg exercises, as well as walking drills. Exercises were individually tailored to address each patient's specific requirements and adapted as required by an exercise scientist. A video demonstration of the home exercises was installed on to the tablet to provide patients with a resource to correctly execute the exercises. Exercise vital sign data (heart rate and oxygen saturation) along with ratings relating to the symptoms of dyspnoea and leg discomfort were recorded by patients immediately after completion of the home exercise programme. These data were transmitted to the web-based platform on three specific days every week for 12 months. The remainder of the data, namely daily steps captured by a pedometer, spirometry, oximetry and responses to questionnaires (Health-related Quality of Life (HRQoL), COPD assessment questionnaire (CAT), Hospital Anxiety and Depression Scale (HADS), modified Medical Research Council dyspnoea scale (mMRC)), were recorded and transmitted twice weekly for 12 months. Patients were asked to complete the HADS questionnaire once every month. Data were transmitted from the tablet to a secure web-based server platform (TELECARE, version 2.2.13, Linkcare Health Services SL, Barcelona, Spain; incorporated and adapted by Singularlogic Integrator S.A., Athens, Greece) [20]. Data were stored on the web-based platform and reviewed regularly (three or four times per week) by the different healthcare professionals (further details in supplementary material).

Hospital-based, outpatient, maintenance rehabilitation programme (group B)

Patients assigned to the hospital-based PR programme visited the hospital twice weekly for 12 months to participate in a multidisciplinary maintenance rehabilitation programme including exercise training, physiotherapy, dietary and psychological advice (supplementary material) [18]. The hospital-based maintenance rehabilitation programme consisted of 96 sessions performed over 12 months.

\section{Usual care}

Usual care included optimal pharmacotherapy oxygen therapy in the presence of respiratory failure, vaccination for Streptococcus pneumoniae, annual vaccination for influenza, and regular follow-up by a respiratory physician according to the guidelines [19]. Furthermore, patients were trained on the early recognition of an acute exacerbation of COPD so that they could seek timely medical care.

\section{Study procedures}

For groups A and B outcome assessment was conducted at baseline, immediately after completion of the primary 2-month PR programme and 12 months later. For group C, outcomes were assessed at the corresponding time points as groups A and B (figure 1). The primary end-point was the rate of moderate to severe acute exacerbation of COPD, hospitalisations because of acute exacerbation of COPD and ED visits, which were compared among the three groups over a period of 12 months following completion of the primary 2-month period. Additional analyses included the rate of severe exacerbations (hospitalisations) and the rate of ED visits because of acute exacerbation of COPD that did not require hospital admission. Decision for hospital admission was based on the judgment of the consultant physicians in accordance with the criteria suggested in the GOLD document [19], including the marked increase in symptom intensity (e.g. sudden development of resting dyspnoea), severe underlying COPD, onset of new physical signs (e.g. cyanosis, peripheral oedema), failure of an exacerbation to respond to initial medical management, presence of serious comorbidities (e.g. heart failure or new arrhythmias), frequent exacerbations, older age and insufficient home support [19]. In regards to the ED visits, they were based on the patients' judgement and the availability of care at the timing of the worsening of the symptoms. Secondary end-points included 1) functional capacity, 2) daily physical activity and 3) health-related quality of life outcomes.

\section{Definitions of acute COPD exacerbation}

Acute exacerbations of COPD were defined according to the GOLD definition [19] as acute events characterised by a worsening of the patient's respiratory symptoms that is beyond normal day-to-day variations and led to a change in medication. Moderate to severe acute exacerbation of COPD were events where patients received antibiotics, systemic corticosteroids or both. Hospitalisations (severe exacerbations) and ED visits because of acute exacerbation of COPD were also assessed. A respiratory physician with expertise in COPD (acted as case manager) was able to recognise symptom deterioration using the study web-based platform and communicated with the patients assigned to the home-based maintenance tele-rehabilitation group when needed. Patients in the other two groups (hospital-based, outpatient, maintenance and usual care) received training to recognise the onset of an acute exacerbation of COPD and contact the study chest physicians in case of symptom deterioration. Monthly telephone contacts with the patients in all groups ensured that no acute exacerbation of COPD event was missing. If patients needed hospital admission the study medical case manager was able to contact the patients' physicians and get all information needed for the patient and the type of acute exacerbation of COPD. Drug prescription could be found in the electronic prescription system used by the Greek medical registration system. 


\section{Outcome measures}

Lung function and functional capacity assessment

Post-bronchodilation dynamic spirometry, diffusing capacity of the lung and evaluation of static lung volumes (supplementary material) [21] were assessed. Incremental exercise tests were performed on an electronic ergometer bicycle with breath-by-breath gas exchange measurements and cardiac output recordings using impedance cardiography to the limit of tolerance (supplementary material). Functional capacity was assessed by the 6-min walk test (6MWT) [22].

\section{Daily physical activity}

Daily physical activity measurements were performed by using a patient activity monitor (Actigraph GT3X, Actilife, Pensacola, FL) validated for COPD [23, 24] (supplementary material).

\section{Health-related quality of life and respiratory symptoms}

HRQoL and respiratory symptoms were evaluated by the following questionnaires [1, 19]: 1) St George's Hospital Respiratory Questionnaire (SGRQ), 2) CAT and 3) mMRC.

\section{Analysis of adherence rates (compliance)}

Adherence to the home-based maintenance tele-rehabilitation and hospital-based, outpatient, maintenance programmes were assessed by the adherence rate (actual number of sessions/total expected number of session $\times 100$ ). Adherence to measurements of vital signs, home exercises, responses to questionnaires and daily steps were recorded for each participant by the number of registrations entered divided by the number of those recommended (more details in supplementary material).

\section{Sample size calculations}

The calculation of sample size was based on ANOVA repeated measurements between the three groups. The minimum detectable difference in the number of hospitalisations for acute exacerbation of COPD was used for the power calculation, which was obtained from a previous study [25]. This previous work evaluated the effect of a PR programme on the frequency of hospitalisation during 1 year after completion of the initial PR programme compared with 1 year before PR. An effect size of 0.42 , based on a mean difference (1.37) and SD (3.26) of acute exacerbations of COPD per year [25], estimated a sample size of 116 patients was required to achieve a power of 0.90 using an alpha significance level of 0.05 (two-sided). To compensate for a potential dropout rate of $20 \%$, a total sample size of 138 patients (46 patients in each group) was determined to be sufficient. Sample size calculation was performed by GPower 3.1.7 software. During the 2-month primary PR programme, three patients from group A were discontinued from the study because of transport barriers.

\section{Statistical analysis}

The Shapiro-Wilk test revealed that all data were normally distributed. One-way ANOVA was utilised to detect differences among the three groups at baseline for all variables. Group differences were investigated by two-way ANOVA with repeated measurements at different time points, namely baseline, 2 months and 14 months after patient enrolment to the study. Where necessary, significance differences were followed up with pair-wise Tukey's post hoc analyses. Time to first acute exacerbation of COPD, hospitalisation for acute exacerbation of COPD and ED visit for each group were evaluated by Kaplan-Meier survival curves and log-rank tests. Poisson regression univariate and multivariate analyses were performed to evaluate the influence of the participation in hospital-based, outpatient, PR or home-based tele-rehabilitation programmes, acute exacerbation of COPD, hospitalisations for acute exacerbation of COPD and visits to the emergency department (ED) in the 1-year of follow-up. Results are presented as hazard ratios (HR) with $95 \%$ confidence intervals. Poisson regression univariate and multivariate analyses were additionally performed to account for variability in exacerbation rates between patients. Skewed data were logarithmically transformed for regression analyses. p-values $\leqslant 0.05$ were considered statistically significant. Data were analysed using SPSS 22.0 for Windows (SPSS Inc., Chicago, IL, USA).

\section{Results}

Acute exacerbations of COPD, hospitalisations and ED visits

Patients' demographic and clinical characteristics at baseline are shown in table 1. Both the home-based maintenance tele-rehabilitation group and the hospital-based group showed a lower rate $(\mathrm{p}<0.001)$ of acute exacerbation of COPD and hospitalisations for acute exacerbation of COPD in the 12 months of follow-up than the usual care group (mean \pm SD $1.7 \pm 1.7$ versus $1.8 \pm 1.4$ versus $3.5 \pm 1.8$; respectively). Similarly, the rates of hospitalisations for acute exacerbation of COPD were lower in the home-based tele-rehabilitation group and the hospital-based group than usual care $(0.3 \pm 0.7,0.3 \pm 0.6$ versus $1.2 \pm 1.7$, respectively, $\mathrm{p}<0.001)$. Finally, the home-based tele-rehabilitation group showed a lower rate of ED visits in 
TABLE 1 Patients' demographic and clinical characteristics at baseline

\begin{tabular}{|c|c|c|c|}
\hline & $\begin{array}{c}\text { Group A } \\
(n=47)\end{array}$ & $\begin{array}{c}\text { Group B } \\
(n=50)\end{array}$ & $\begin{array}{c}\text { Group C } \\
(n=50)\end{array}$ \\
\hline Men/women $\mathrm{n}$ & $44 / 3$ & $38 / 12$ & $37 / 13$ \\
\hline Age years & $66.9 \pm 9.6$ & $66.7 \pm 7.3$ & $64.0 \pm 8.0$ \\
\hline FEV 1 L & $1.55 \pm 0.80$ & $1.41 \pm 0.48$ & $1.42 \pm 0.66$ \\
\hline FEV $1 \%$ pred & $49.6 \pm 21.9$ & $51.8 \pm 17.3$ & $51.7 \pm 21.0$ \\
\hline FVC L & $3.07 \pm 0.90$ & $2.70 \pm 0.65$ & $2.77 \pm 0.81$ \\
\hline FVC \% pred & $80.7 \pm 20.2$ & $78.4 \pm 18.4$ & $80.0 \pm 20.3$ \\
\hline FEV $1 /$ FVC \% & $47.0 \pm 14.1$ & $49.0 \pm 12.7$ & $51.9 \pm 12.4$ \\
\hline VC $\%$ pred & $83.6 \pm 20.6$ & $85.5 \pm 14.6$ & $82.6 \pm 21.9$ \\
\hline IC \% pred & $81.7 \pm 33.0$ & $77.3 \pm 30.0$ & $76.9 \pm 31.0$ \\
\hline TLC \% pred & $118.8 \pm 30.3$ & $120.7 \pm 25.7$ & $119.9 \pm 28.8$ \\
\hline FRC $\%$ pred & $158.5 \pm 60.5$ & $154.5 \pm 43.0$ & $154.4 \pm 56.6$ \\
\hline RV \% pred & $184.6 \pm 80.6$ & $180.2 \pm 59.9$ & $182.0 \pm 70.9$ \\
\hline DLco $\%$ pred & $53.5 \pm 19.9$ & $57.0 \pm 20.4$ & $55.9 \pm 28.4$ \\
\hline $\mathrm{SpO}_{2} \%$ & $93.0 \pm 3.0$ & $94.0 \pm 2.9$ & $94.0 \pm 3.0$ \\
\hline 6MWT m & $389.1 \pm 91.3$ & $385.1 \pm 80.3$ & $384.8 \pm 80.2$ \\
\hline Body mass index $\mathrm{kg} \cdot \mathrm{m}^{-2}$ & $28.0 \pm 5.3$ & $27.5 \pm 5.0$ & $26.4 \pm 5.0$ \\
\hline BODE index & $3.5 \pm 2.7$ & $3.2 \pm 2.1$ & $3.3 \pm 2.3$ \\
\hline mMRC & $2.3 \pm 1.0$ & $2.5 \pm 1.0$ & $2.2 \pm 1.1$ \\
\hline Oxygen therapy (LTOT) n & 13 & 9 & 15 \\
\hline Smoking status (cur. versus ex.) n & $7 / 40$ & $4 / 46$ & $3 / 47$ \\
\hline \multicolumn{4}{|l|}{ Drug therapy $\mathrm{n}$} \\
\hline LAMA & 36 & 37 & 35 \\
\hline LABA & 22 & 26 & 24 \\
\hline ICS & 32 & 37 & 41 \\
\hline \multicolumn{4}{|l|}{ At least one comorbidity } \\
\hline Cardiovascular $\mathrm{n}$ & 14 & 15 & 13 \\
\hline Number of acute COPD exacerbations & $3.3 \pm 1.3$ & $3.4 \pm 1.4$ & $3.3 \pm 1.6$ \\
\hline
\end{tabular}

Values are presented as mean $\pm S D$, unless otherwise stated. $F E V 1$ : forced expiratory volume in $1 \mathrm{~s} ; \mathrm{FVC}$ : forced vital capacity; VC: vital capacity; IC: inspiratory capacity; TLC: total lung capacity; FRC: functional residual capacity; $\mathrm{RV}$ : reserve volume; $\mathrm{DLCO}$ : diffusion capacity of the lung for carbon monoxide; $\mathrm{SpO}_{2}$ : oxygen saturation measured by pulse oximetry; 6MWT: 6-min walk test; mMRC: modified Medical Research Council dyspnoea scale; LTOT: long-term oxygen therapy; LAMA: long-acting muscarinic agonist; LABA: long-acting beta-agonist; ICS: inhaled corticosteroid; group A: home-based maintenance tele-rehabilitation; group B: hospital-based, outpatient, maintenance rehabilitation; group C: usual care.

the 12 months of follow-up than both the hospital-based group and the usual care group ( $0.5 \pm 0.9$ versus $1.8 \pm 1.5$ versus $3.5 \pm 1.8$, respectively; $\mathrm{p}<0.001)$.

Kaplan-Meier survival curves evaluating the time to next acute exacerbation of COPD and hospitalisations for acute exacerbation of COPD in the 12 months of follow-up are presented in figure 2 . In the univariate Poisson regression analysis, significant predictors of acute exacerbation of COPD were smoking status, FEV1 (\% predicted), number of acute exacerbations of COPD in the preceding year and PR (either home-based maintenance tele-rehabilitation or hospital-based, outpatient, maintenance rehabilitation; table 2). Accordingly, significant predictors of hospitalisations for acute exacerbation of COPD were gender, FEV1 (\% predicted), number of acute exacerbations of COPD in the preceding year, and PR (either hospital-based, outpatient, maintenance PR or home-based maintenance tele-rehabilitation; table 2). In multivariate analysis, PR (both home-based tele-rehabilitation and hospital-based, outpatient rehabilitation) remained an independent predictor of a lower risk for acute exacerbation of COPD in the 12-month follow up (incidence rate ratio (IRR) $0.517,95 \%$ CI $0.389-0.687 ; \mathrm{p}<0.001$ ) and (IRR 0.635, 95\% CI 0.473-0.853; $\mathrm{p}=0.003$ ) for the home-based maintenance tele-rehabilitation and hospital-based, outpatient, maintenance PR groups, respectively. In addition, PR (both home-based maintenance tele-rehabilitation and hospital-based maintenance rehabilitation) remained an independent predictor of a lower risk for hospitalisations for acute exacerbation of COPD (IRR 0.189, 95\% CI 0.100-0.358; p<0.001) and (IRR 0.375, 95\% CI 0.207-0.681; $\mathrm{p}=0.001$ ) for home-based maintenance tele-rehabilitation and hospital-based maintenance PR, respectively. However, only home-based maintenance tele-rehabilitation and not hospital-based maintenance PR was an independent predictor of visits in the ED (IRR 0.116, 95\% CI 0.072-0.185; p<0.001) (table 2). 

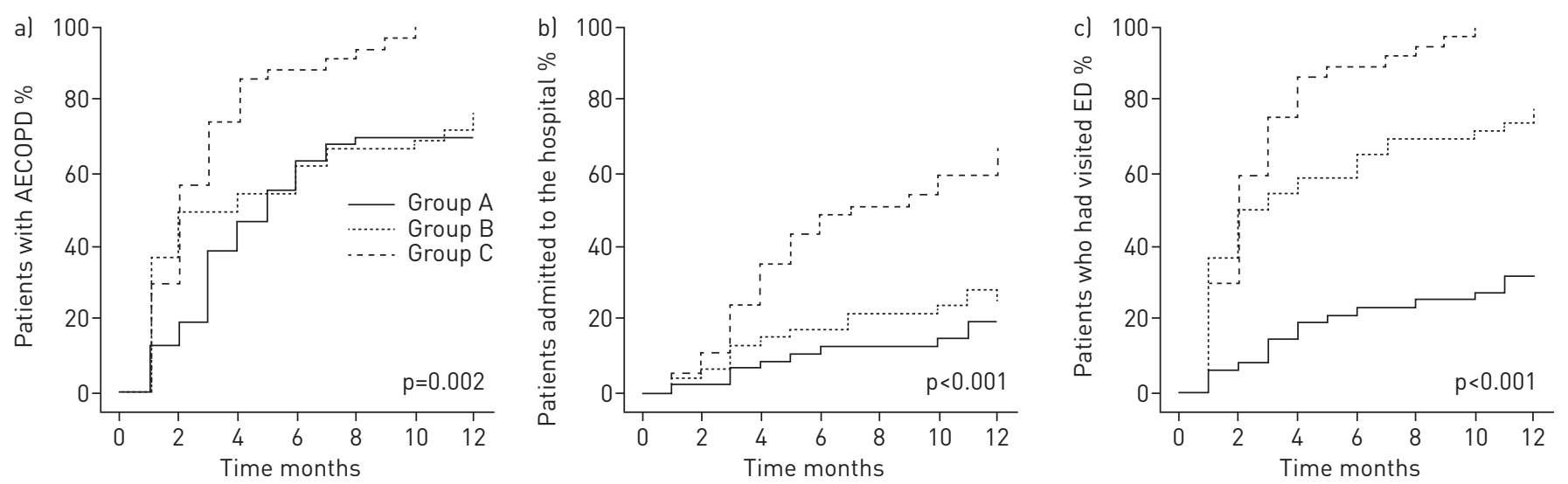

FIGURE 2 Percentage of patients with acute exacerbations (AE) of chronic obstructive pulmonary disease (COPD) (a) hospitalisations (b) and emergency department (ED) visits (c) during the 12 months follow up. Group A: home-based maintenance tele-rehabilitation; group B: hospital-based, outpatient, rehabilitation; and group C: usual care treatment. Note that acute exacerbations of COPD refers to all types of acute exacerbations of COPD, regardless of the place where they were treated (home, hospital or ED).

\section{Functional capacity}

Home-based maintenance tele-rehabilitation was equally effective for hospital-based, outpatient, maintenance PR in preserving for 12 months the initial statistical and/or clinically meaningful improvements in peak work rate $(\mathrm{p}=0.011)$ and the distance covered during the 6MWT, respectively, and were superior to usual care (table 3). The magnitude of improvement induced by the primary 2-month PR programme in the aforementioned variables was not different between groups A and B. Following the initial PR programme the

TABLE 2 Predictors of acute exacerbations of chronic obstructive pulmonary disease (COPD), hospitalisations and emergency department (ED) visits

\begin{tabular}{|c|c|c|c|c|c|c|}
\hline & \multicolumn{3}{|c|}{ Univariate analysis } & \multicolumn{3}{|c|}{ Multivariate analysis } \\
\hline & IRR & $95 \% \mathrm{Cl}$ & p-value & IRR & $95 \% \mathrm{Cl}$ & p-value \\
\hline \multicolumn{7}{|l|}{ Acute exacerbations of COPD } \\
\hline Gender (female) & 1.225 & $0.942-1.595$ & 0.131 & & & \\
\hline Smoking status (ex-smoker) & 1.727 & $1.170-2.550$ & 0.006 & 1.045 & $0.694-1.573$ & 0.833 \\
\hline FEV $1 \%$ pred $)$ & 0.985 & $0.979-9.991$ & $<0.001$ & 0.992 & $0.986-0.999$ & 0.016 \\
\hline Group B & 0.498 & $0.375-0.660$ & $<0.001$ & 0.635 & $0.473-0.853$ & 0.003 \\
\hline \multicolumn{7}{|l|}{ Group C (indicator) } \\
\hline \multicolumn{7}{|l|}{ Hospitalisations for acute exacerbations of COPD } \\
\hline Age & 1.002 & $0.975-1.030$ & 0.877 & & & \\
\hline Gender (female) & 0.497 & $0.248-0.997$ & 0.049 & 0.475 & $0.208-0.871$ & 0.019 \\
\hline Smoking status (ex-smoker) & 1.523 & $0.732-3.170$ & 0.261 & & & \\
\hline \multicolumn{7}{|l|}{ Group C (indicator) } \\
\hline \multicolumn{7}{|l|}{ ED visits for acute exacerbations of COPD } \\
\hline Age & 0.990 & $0.975-1.005$ & 0.191 & & & \\
\hline Gender (female) & 1.536 & $1.162-2.031$ & 0.003 & 1.051 & $0.772-1.431$ & 0.752 \\
\hline Smoking status (ex-smoker) & 1.964 & $1.242-3.105$ & 0.004 & 0.815 & $0.504-1.318$ & 0.404 \\
\hline FEV 1 (\% pred $)$ & 0.987 & $0.980-0.994$ & $<0.001$ & 0.992 & $0.984-0.994$ & 0.027 \\
\hline Acute exacerbations of COPD in preceding year & 1.156 & $1.113-1.201$ & $<0.001$ & 1.244 & $1.175-1.316$ & $<0.001$ \\
\hline Group A & 0.151 & $0.099-0.232$ & $<0.001$ & 0.116 & $0.072-0.185$ & $<0.001$ \\
\hline Group B & 0.501 & $0.380-0.661$ & $<0.001$ & 0.750 & $0.555-1.015$ & 0.062 \\
\hline Group C (indicator) & & & & & & \\
\hline
\end{tabular}

IRR: incidence rate ratio; FEV1: forced expiratory volume in $1 \mathrm{~s}$; group A: home-based maintenance tele-rehabilitation; group B: hospital-based, outpatient, maintenance pulmonary rehabilitation; group C: usual care. 


\begin{tabular}{|c|c|c|c|}
\hline & Baseline & 2 months & 14 months \\
\hline \multicolumn{4}{|c|}{ Peak work rate W } \\
\hline Group A & $63 \pm 33$ & $74 \pm 35^{\#}$ & $76 \pm 35^{\#}$ \\
\hline Group B & $67 \pm 25$ & $79 \pm 32^{\#}$ & $79 \pm 31^{\#}$ \\
\hline Group C & $65 \pm 24$ & $63 \pm 27$ & $58 \pm 24^{\#}$ \\
\hline \multicolumn{4}{|l|}{ 6MWT m } \\
\hline Group A & $389.1 \pm 91.3$ & $422.1 \pm 70.5^{\pi}$ & $420.2 \pm 74.9^{\pi}$ \\
\hline Group B & $385.1 \pm 80.3$ & $423.0 \pm 70.5^{\Uparrow}$ & $427.5 \pm 63.0^{9}$ \\
\hline Group C & $384.8 \pm 80.2$ & $382.4 \pm 80.3$ & $339.9 \pm 110.1^{9}$ \\
\hline
\end{tabular}

Values are presented as mean \pm SD. 6MWT: 6-min walking test; group A: home-based maintenance tele-rehabilitation; group B: hospital-based, outpatient, maintenance rehabilitation; group C: usual care.

\#: statistically significant differences from baseline; " : clinically meaningful differences from baseline.

fraction of patients who improved beyond the minimal clinically important difference $(>25 \mathrm{~m})$ the $6 \mathrm{MWT}$ was for group A: $53 \%$, group B: $60 \%$ and group C: $4 \%$.

\section{HRQoL, respiratory symptoms and chronic dyspnoea}

Home-based maintenance tele-rehabilitation was equally effective as hospital-based, outpatient, maintenance rehabilitation in preserving the initial clinically meaningful improvement in SGRQ, CAT and mMRC scores over a period of 12 months and was superior to usual care, exhibiting deterioration in the questionnaire scores over the same period (table 4). The magnitude of improvement induced by the primary 2-month PR programme in the aforementioned parameters was not different between the two rehabilitation groups.

\section{Daily physical activity}

Home-based maintenance tele-rehabilitation was equally effective to hospital-based maintenance, outpatient, PR in preserving the initial improvement in time spent in sedentary, light, lifestyle and moderate daily physical activities over the 12-month period, and was superior to usual care exhibiting an increase in time spent in sedentary, and decrease in lifestyle, and moderate daily activities over 12 months of follow-up (figure 3 and supplementary material).

\section{Adherence/compliance}

The overall compliance to the different components of the home-based maintenance tele-rehabilitation intervention (figure 4 and supplementary material) over 12 months of follow-up was $93.5 \%$.

\begin{tabular}{|c|c|c|c|}
\hline & Baseline & 2 months & 14 months \\
\hline \multicolumn{4}{|l|}{ SGRQ } \\
\hline Group A & $46.2 \pm 19.7$ & $42.2 \pm 19.2^{n}$ & $38.4 \pm 20.5^{\pi}$ \\
\hline Group B & $43.5 \pm 16.7$ & $35.4 \pm 15.7^{\text {ๆ }}$ & $33.6 \pm 16.5^{\pi}$ \\
\hline Group C & $44.1 \pm 16.6$ & $44.7 \pm 16.9$ & $50.2 \pm 17.7^{\pi}$ \\
\hline \multicolumn{4}{|l|}{ CAT } \\
\hline Group A & $17.6 \pm 8.1$ & $12.9 \pm 7.5^{\pi}$ & $13.0 \pm 7.3^{\Uparrow}$ \\
\hline Group B & $15.7 \pm 5.6$ & $13.2 \pm 5.8^{\mathbb{1}}$ & $11.8 \pm 5.6^{9}$ \\
\hline Group C & $15.8 \pm 4.9$ & $16.1 \pm 6.2$ & $20.9 \pm 6.7^{\uparrow}$ \\
\hline \multicolumn{4}{|l|}{ mMRC } \\
\hline Group A & $2.3 \pm 1.0$ & $1.8 \pm 0.9^{\text {व }}$ & $1.6 \pm 1.0^{9}$ \\
\hline Group B & $2.5 \pm 1.0$ & $1.5 \pm 0.9^{9}$ & $1.3 \pm 0.9$ ก \\
\hline Group C & $2.2 \pm 1.1$ & $2.5 \pm 1.0$ & $3.1 \pm 0.8^{\text {ๆ }}$ \\
\hline
\end{tabular}

Values are presented as mean \pm SD. SGRQ: St George's Respiratory Questionnaire; CAT: COPD Assessment Test; mMRC: modified Medical Research Council dyspnoea scale; group A: home-based maintenance tele-rehabilitation; group B: hospital-based, outpatient, maintenance rehabilitation; group C: usual care. १: clinically meaningful differences from baseline. 

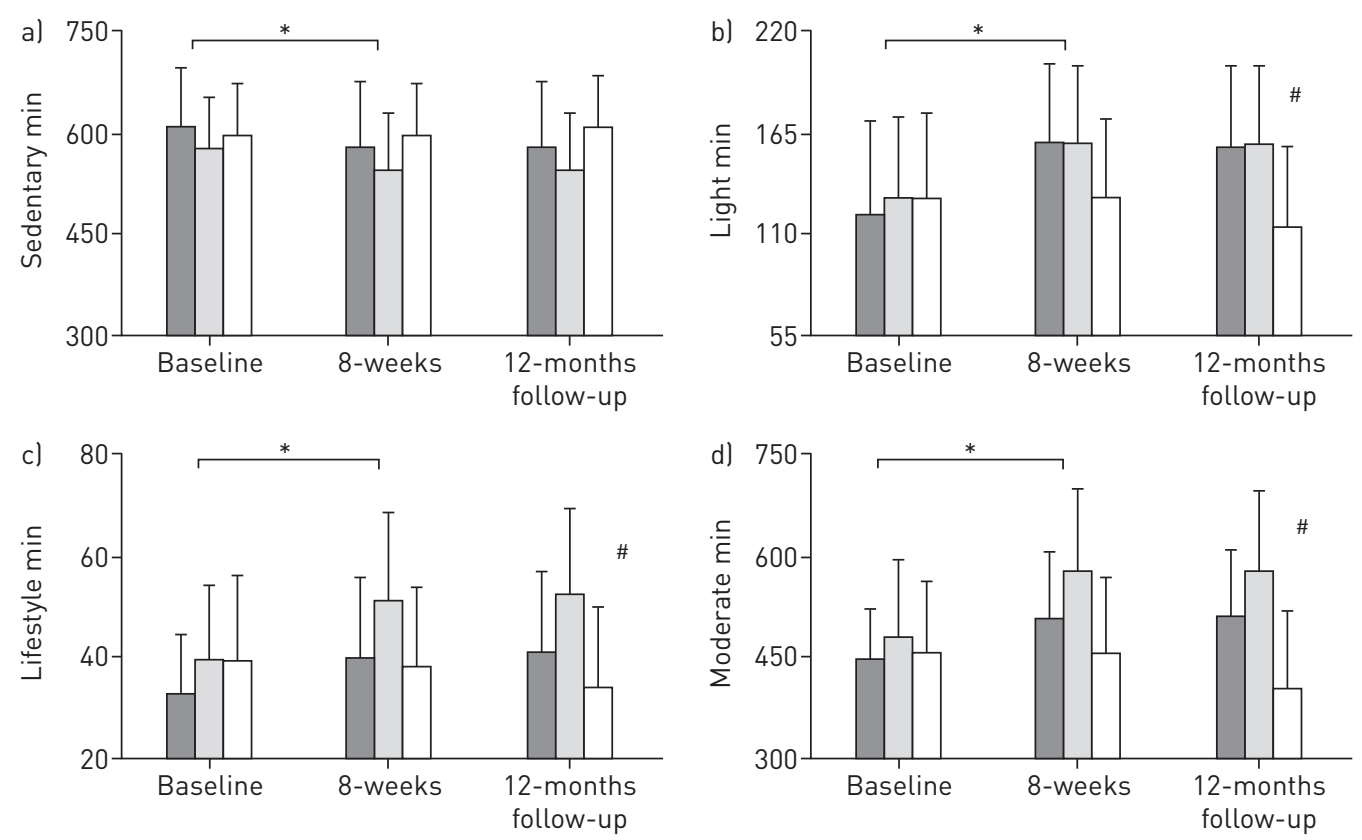

FIGURE 3 Changes in daily physical activity levels defined by the time spent in sedentary (a), light (b), lifestyle (c) and moderate (d) activities. Values are mean $\pm \mathrm{SD}$. *: statistically significant differences from baseline for the home-based maintenance tele-rehabilitation (dark grey bars) and hospital-based, outpatient, rehabilitation (light grey bars) groups. \#: statistically significant differences from the 2-month time point for the usual care group (white bars).

\section{Discussion}

The main finding of the study was that home-based maintenance tele-rehabilitation via monitoring of vital signs was as effective as hospital-based, outpatient, maintenance PR and superior to usual care in terms of reducing the risk for acute exacerbation of COPD and hospitalisations, while preserving the functional and HRQoL benefits of a primary PR programme over a period of 12 months. Moreover, only home-based maintenance tele-rehabilitation and not hospital-based, outpatient, maintenance PR was an independent predictor of reduced risk for ED visits.

Recently, an international task force commissioned by the ATS and ERS delivered policy recommendations to improve access and delivery of PR services to suitable patients [8]. It was recognised that although traditional

FIGURE 4 Patients' compliance to the different components of monitoring and home-based maintenance telerehabilitation maintenance programme over a 12-month period (group A, $n=47)$.

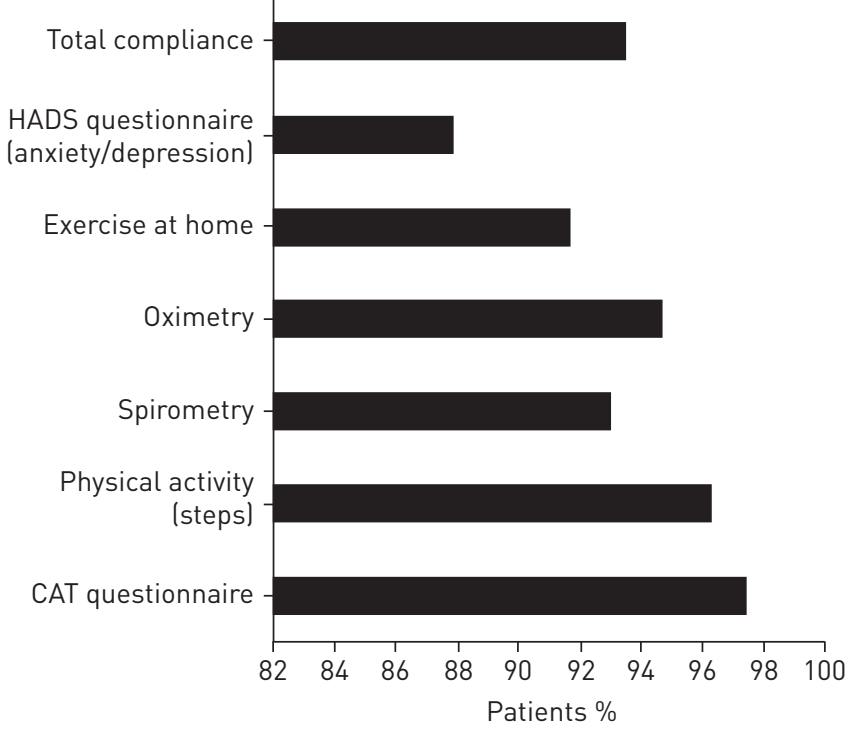


models of outpatient PR are suitable for many patients, several barriers prevent the vast majority of eligible patients from accessing or adhering to these programmes, particularly in regions or healthcare systems where traditional models of PR are not feasible [8]. It was, thus, recommended that research should focus on the effectiveness of alternative models of PR such as tele-rehabilitation [26].

In the present study we have included a population of high-risk patients with a history of exacerbations, as the majority of them experienced ED visits and hospitalisations during the study, in accordance with previous observations in similar populations in Greece [47]. Our findings on the reduction of the risk for acute exacerbation of COPD for both home-based maintenance tele-rehabilitation and hospital-based maintenance strategies are consistent with previous randomised controlled trials (RCTs) [27, 28] employing comparable protocols of initially intense PR programmes followed by home maintenance sessions. In addition, our results on the reduction of risk for hospitalisations for acute exacerbation of COPD concur with those [27-29] applying long-term follow-up maintenance strategies. Importantly, our findings on the reduction of the overall rate of hospitalisations for acute exacerbation of COPD per person per year at risk for the home-based maintenance tele-rehabilitation group (by 0.19 , 95\% CI 0.10-0.35) and the hospital-based, outpatient, maintenance programme (by $0.38,95 \%$ CI $0.21-0.68$ ) compare favourably with the overall rate recently reported form the meta-analysis of 10 RCT studies (by $0.62,95 \%$ CI $0.33-1.16$ ) [7]. Furthermore, the effectiveness of home tele-rehabilitation in reducing the risk for ED visits provides evidence that early patient and physician recognition of acute exacerbation of COPD followed by prompt treatment initiation prevent COPD-related health worsening [27-30]. The home-based tele-rehabilitation group had the advantage of spirometry and physical signs monitoring that may have further supported the early recognition of acute exacerbation of COPD. Indeed, a more prompt recognition of symptom and/or lung function deterioration may have resulted in the prompt intervention for medical care and decreased the need for an ED visit.

The economic burden of COPD increases with increasing disease severity, and it is substantially augmented by exacerbations and hospitalisations. A study conducted in Greece has estimated that the mean cost per severe COPD exacerbation is $€ 1711$ (range $€ 1357-€ 2614$ ), depending on the severity [31]. These findings highlight the importance of reducing the frequency of acute exacerbation of COPD and hospitalisations. This is particularly important in light of findings indicating that a significant proportion of COPD patients in Greece experience two or more acute exacerbations of COPD per year and that the majority of these exacerbations are moderate or severe, frequently leading to hospitalisations [32]. Collectively, these findings emphasise the importance of decreasing the number of acute exacerbations of COPD per patient per year to alleviate the disease burden and the economic cost of the disease. In regards to cost savings for delivering the present home-based maintenance tele-rehabilitation programme over a period of 12 months, the calculated total cost per patient including equipment, development of the ICT platform, use of $3 \mathrm{G}$ network and cost for personnel was approximately $€ 1800$. This figure is equivalent to approximately $60 \%$ of the total estimated cost $(€ 2908)$ spared by reducing the frequency of acute exacerbations of COPD (by 1.7 exacerbations per patient per year $€ 1711=€ 2908$ ) [31]. In addition, this figure is approximately $40 \%$ of the estimated cost for 1 year of hospital-based maintenance rehabilitation sessions including twice-weekly outpatient respiratory department visits.

Remote monitoring of vital signs allows clinicians to monitor a patient remotely with reference to physiological signs, respiratory symptoms and activity levels using a wide range of technological devices [10,33-36]. In this study, we have implemented patient tele-consultation based on vital sign data (recorded by patients using portable devices) transmitted to an ICT web based platform via patients' tablets. In addition, patients were provided with feedback to maintain progress in respect to executing home exercise drills and enhancing daily activity levels. Accordingly, we have described a novel home-based maintenance tele-rehabilitation approach to reduce the risk for acute exacerbation of COPD and hospitalisations for acute exacerbation of COPD to a similar extent of a hospital-based maintenance rehabilitation programme.

The home-based maintenance tele-rehabilitation programme was not by any means inferior to the supervised hospital-based, outpatient, maintenance PR programme in preserving true physiological training effects, respiratory symptoms, daily activity levels and aspects of quality of life over a period of 12 months. Previous studies have also reported positive effects of hospital-based maintenance PR programmes on exercise capacity and quality of life [6]. However, insufficient funding, resources and reimbursement limit the implementation of such hospital-based programmes worldwide [8]. To the best of our knowledge, this is the first study to show that home-based rehabilitation with the use of ICT is effective in preserving the long-term physiological training effects initially acquired by a comprehensive hospital-based PR programme. Nevertheless, future studies by healthcare professionals are required to inform and disseminate information on the costs, clinicaland cost-effectiveness of maintenance rehabilitation programmes to payers [8]. Patients assigned to the usual care group not undertaking the initial 2-month outpatient PR programme and subsequently the 12-month maintenance programme experienced significant deterioration in functional capacity and quality of life, further highlighting the beneficial effects of PR maintenance strategies [27-29]. 
The finding that home-based maintenance tele-rehabilitation preserved the initial physiological benefits deserves further analysis to justify these findings. Potential factors may include [36] 1) excellent patient adherence to regular vital sign recordings (figure 4), 2) very good compliance to home exercise drills, 3) patient empowerment in the promotion of high levels of daily physical activity, 4) regular feedback on patient's progress through motivation messages to tablets or via telephone contacts and 5) adherence to sufficient exercise training loads and self-management techniques. Indeed, regular investigator-patient communications facilitated their empowerment in the promotion of increased levels of physical activity and raised their awareness to comply with the measurement and physical activity requirements of the protocol.

Incomplete implementation of the aforementioned factors and inadequate sample size power [37-39] most likely explains why previously implemented tele-rehabilitation studies did not demonstrate such long-term benefits [38, 40,41]. Combining self-management techniques, regular exercise training and contact with the PR personnel is likely to have contributed to reinforcing behavioural changes towards a more active lifestyle [42]. Our findings support this notion since the combination of home physical training, physical activity consultation and personalised feedback on weekly physical activity levels decreased the time spent in sedentary activities and improved the time spent in light and moderate lifestyle activities [43]. Given that low levels of daily physical activity is an independent risk factor for acute exacerbation of COPD [42], it is plausible that maintenance of enhanced daily physical activity over a period of 12 months could have contributed to the reported reduced risk for acute exacerbation of COPD. This is an important outcome since earlier studies including a recent systematic review with meta-analyses [15-17] highlight the finding that tele-healthcare interventions have minimal impact on patients' physically active time.

Our findings are in tandem with those of HoAas et al. [13], who showed that following completion of an initial (4 week) supervised PR programme, improvements in 6MWT and quality of life were preserved for 1 year when a home tele-rehabilitation programme was implemented. These outcomes are justified by the high adherence rate for vital sign measurements and training sessions. Indeed, our multimodal home-based tele-rehabilitation and tele-consultation programme, in contrast to other studies [36, 44], resulted in very good adherence without dropouts over a period of 12 months.

\section{Study limitations}

Our study design was not blinded, and as such the investigators were aware of the allocation of patients into the different maintenance rehabilitation groups. Importantly, patients were given general information about their participation in the study and details on the interventions related only to their intervention arm. Moreover, the choice of objective endpoints that were related to healthcare resource use (moderate or severe acute exacerbations of COPD, hospitalisations and ED visits) minimises to the best possible extent potential biases.

Another potential limitation of the study is that the usual care group (C) of our study did not participate in a PR programme. PR in Greece is delivered only by a few University Medical Departments. Hence, the majority of COPD patients follow usual care only, which unfortunately does not include access to PR. This is due to the lack of specialised rehabilitation centres and trained personnel. This is why our study was designed to represent the reality in the local community and provide evidence to health authorities of the major advantages of PR so that an infrastructure (personnel and resources) can be allocated to establish PR programmes in the community or at home across the nation. Lack of access for the usual care group to the initial 2-month PR programme may therefore constitute a limitation as one could argue that maintenance of benefits might be, at least in part, related to the conduction of initial PR, which brought benefits to only those patients who undertook PR. However, it is well documented that benefits of PR are often lost over a period of 12 months without implementation of a maintenance strategy [1-6]. Hence, an important aspect of our study was to identify the best maintenance strategy to preserve the initial benefits of PR for an extended period.

The hospital-based, outpatient, maintenance programme was conducted at the hospital's gymnasium using specialised equipment (e.g. stationary bicycles and weight lifting apparatus), whereas the home-based exercise programme comprised arm and leg exercises, as well as walking drills without the use of any specialised equipment. This is the reason why we offered three weekly sessions at home (as opposed to two sessions during the outpatient hospital-based maintenance programme) in an attempt to ensure adequate weekly physical exercise in this group. This difference in the total number of maintenance sessions between the two maintenance strategies over the 12-month follow-up period could have potentially introduced bias. Nevertheless, our finding that the home-based maintenance tele-rehabilitation programme was equally effective as the hospital-based, outpatient, maintenance PR in preserving for 12 months the initial statistical and/or clinically meaningful improvements in peak work rate and the 6MWT, respectively, suggest that the applied overall training load was well matched between the hospital-based and the home-based groups.

The incidence of acute exacerbation of COPD is often related to multiple factors, including appropriate care by the attending physicians, adherence to treatment $[45,46]$, and comorbidities that include anxiety 
and depression [47] and cardiovascular comorbidities [48]. However, we have attempted to delimit the impact of such confounding factors during the study, as all patients were prescribed optimal treatment for COPD and co-existent conditions, received appropriate training on the use of inhaled medication and were optimally followed-up by respiratory physicians.

\section{Clinical implications}

Application of home-based maintenance tele-rehabilitation programmes may lead to significant reductions of healthcare resource use for patients with COPD compared with usual care, with potential benefits on patients' outcomes and quality of life.

\section{Conclusions}

Home-based maintenance tele-rehabilitation is equally effective as hospital-based, outpatient, maintenance $\mathrm{PR}$ in reducing the risk for acute exacerbations of COPD and hospitalisations and encounters a lower risk for ED visits, thereby potentially constituting an effective alternative strategy to hospital-based, outpatient, maintenance rehabilitation.

\section{Acknowledgements}

We are grateful to the collaborators (www.telecare.net.gr) who provided technological assistance for the development of the "TELECARE" Medical platform (J.M. Sanchez and Jim Roldan from Linkcare Health Services SL and O. Kocsis, A. Tsopanoglou, S. Pantenopoulos, from Singularlogic S.A.), as well as the healthcare personnel in charge of provision of dietary advice (A. Thomopoulou), psychological support (C. Feridou) and physical conditioning (E. Plataniti).

An account of this work entitled "Effectiveness of home tele-rehabilitation on functional capacity and daily physical activity in COPD patients" was presented at the 25th International Congress of the ERS (Amsterdam, the Netherlands, September 26-30, 2015) and was awarded "Best Abstract" by the Clinical Assembly.

\section{References}

1 Spruit MA, Singh SJ, Garvey C, et al. An official American Thoracic Society/European Respiratory Society statement: key concepts and advances in pulmonary rehabilitation. Am J Respir Crit Care Med 2013; 188: e13-e64.

2 Spruit MA, Singh SJ. Maintenance programs after pulmonary rehabilitation: how may we advance this field? Chest 2013; 144: 1091-1093.

3 Beauchamp MK, Evans R, Janaudis-Ferreira T, et al. Systematic review of supervised exercise programs after pulmonary rehabilitation in individuals with COPD. Chest 2013; 144: 1124-1133.

4 Foglio K, Bianchi L, Ambrosino N. Is it really useful to repeat outpatient pulmonary rehabilitation programs in patients with chronic airway obstruction? A 2-year controlled study. Chest 2001; 119: 1696-1704.

5 Holland AE, Mahal A, Hill CJ, et al. Home-based rehabilitation for COPD using minimal resources: a randomised, controlled equivalence trial. Thorax 2017; 72: 57-65.

6 Maltais F, Bourbeau J, Shapiro S, et al. Effects of home-based pulmonary rehabilitation in patients with chronic obstructive pulmonary disease: a randomized trial. Ann Intern Med 2008; 149: 869-878.

7 Moore E, Palmer T, Newson R, et al. Pulmonary rehabilitation as a mechanism to reduce hospitalizations for acute exacerbations of COPD: a systematic review and meta-analysis. Chest 2016; 150: 837-859.

8 Rochester CL, Vogiatzis I, Holland AE, et al. An official American Thoracic Society/European Respiratory Society policy statement: enhancing implementation, use, and delivery of pulmonary rehabilitation. Am J Respir Crit Care Med 2015; 192: 1373-1386.

9 Brennan D, Tindall L, Theodoros D, et al. A blueprint for telerehabilitation guidelines. Int J Telerehabil 2010; 2: $31-34$.

10 Holland AE, Hill CJ, Rochford P, et al. Telerehabilitation for people with chronic obstructive pulmonary disease: feasibility of a simple, real time model of supervised exercise training. J Telemed Telecare 2013; 19: 222-226.

11 Goldstein RS, O'Hoski S. Telemedicine in COPD: time to pause. Chest 2014; 145: 945-949.

12 Stickland M, Jourdain T, Wong EY, et al. Using Telehealth technology to deliver pulmonary rehabilitation in chronic obstructive pulmonary disease patients. Can Respir J 2011; 18: 216-220.

13 Hoaas $\mathrm{H}$, Andreassen HK, Lien LA, et al. Adherence and factors affecting satisfaction in long-term telerehabilitation for patients with chronic obstructive pulmonary disease: a mixed methods study. BMC Med Inform Decis Mak 2016; 16: 26.

14 Zanaboni $\mathrm{P}$, Hoaas $\mathrm{H}$, Aaroen Lien L, et al. Long-term exercise maintenance in COPD via telerehabilitation: a two-year pilot study. J Telemed Telecare 2017; 23: 74-82.

15 Lundell S, Holmner A, Rehn B, et al. Telehealthcare in COPD: A systematic review and meta-analysis on physical outcomes and dyspnea. Respir Med 2015; 109: 11-26.

16 Tabak M, Brusse-Keizer M, van der Valk P, et al. A telehealth program for self-management of COPD exacerbations and promotion of an active lifestyle: a pilot randomized controlled trial. Int J Chron Obstruct Pulmon Dis 2014; 9: 935-944.

17 Marquis N, Larivee P, Dubois MF, et al. Are improvements maintained after in-home pulmonary telerehabilitation for patients with chronic obstructive pulmonary disease? Int J Telerehabil 2015; 6: 21-30.

18 Vogiatzis I, Terzis G, Nanas S, et al. Skeletal muscle adaptations to interval training in patients with advanced COPD. Chest 2005; 128: 3838-3845.

19 GOLD. Global Strategy for Diagnosis, Management, and Prevention of COPD. 2014. Available from: http://goldcopd.org/

20 Kocsis O, Vasilopoulou M, Tsopanoglou A, et al. Telemonitoring system for home rehabilitation of patients with COPD. The 5th IEEE International Conference on E-Health and Bioengineering - EHB 2015, Iaşi, Romania, 2015. Available from: http://ieeexplore.ieee.org/document/7391438/ 
21 ATS. Standardization of Spirometry, 1994 Update. American Thoracic Society. Am J Respir Crit Care Med 1995; 152: 1107-1136.

22 ATS. ATS statement: guidelines for the six-minute walk test. Am J Respir Crit Care Med 2002; 166: 111-117.

23 Rabinovich RA, Louvaris Z, Raste Y, et al. Validity of physical activity monitors during daily life in patients with COPD. Eur Respir J 2013; 42: 1205-1215.

24 Van Remoortel H, Raste Y, Louvaris Z, et al. Validity of six activity monitors in chronic obstructive pulmonary disease: a comparison with indirect calorimetry. PloS ONE 2012; 7: e39198.

25 van Ranst D, Stoop WA, Meijer JW, et al. Reduction of exacerbation frequency in patients with COPD after participation in a comprehensive pulmonary rehabilitation program. Int J Chron Obstruct Pulmon Dis 2014; 9: 1059-1067.

26 Vogiatzis I, Rochester CL, Spruit MA, et al. Increasing implementation and delivery of pulmonary rehabilitation: key messages from the new ATS/ERS policy statement. Eur Respir J 2016; 47: 1336-1341.

27 Guell R, Casan P, Belda J, et al. Long-term effects of outpatient rehabilitation of COPD: A randomized trial. Chest 2000; 117: 976-983.

28 Rubi M, Renom F, Ramis F, et al. Effectiveness of pulmonary rehabilitation in reducing health resources use in chronic obstructive pulmonary disease. Arch Phys Med Rehabil 2010; 91: 364-368.

29 Behnke M, Jorres RA, Kirsten D, et al. Clinical benefits of a combined hospital and home-based exercise programme over 18 months in patients with severe COPD. Monaldi Arch Chest Dis 2003; 59: 44-51.

30 Achelrod D, Schreyogg J, Stargardt T. Health-economic evaluation of home telemonitoring for COPD in Germany: evidence from a large population-based cohort. Eur J Health Econ 2016; in press [https://doi.org/10. 1007/s10198-016-0834-x].

31 Geitona M, Hatzikou M, Steiropoulos P, et al. The cost of COPD exacerbations: a university hospital-based study in Greece. Respir Med 2011; 105: 402-409.

32 Stafyla E, Kerenidi T, Gourgoulianis KI. Chronic obstructive pulmonary disease exacerbation frequency and severity. Int J Chron Obstruct Pulmon Dis 2013; 8: 533-535.

33 Barberan-Garcia A, Vogiatzis I, Solberg HS, et al. Effects and barriers to deployment of telehealth wellness programs for chronic patients across 3 European countries. Respir Med 2014; 108: 628-637.

34 Lundell S, Holmner A, Rehn B, et al. Telehealthcare in COPD: a systematic review and meta-analysis on physical outcomes and dyspnea. Respir Med 2015; 109: 11-26.

35 Paneroni M, Colombo F, Papalia A, et al. Is Telerehabilitation a safe and viable option for patients with COPD? A feasibility study. COPD 2015; 12: 217-225.

36 Cruz J, Brooks D, Marques A. Home telemonitoring in COPD: a systematic review of methodologies and patients' adherence. Int J Med Inform 2014; 83: 249-263.

37 Bourbeau J, Julien M, Maltais F, et al. Reduction of hospital utilization in patients with chronic obstructive pulmonary disease: a disease-specific self-management intervention. Arch Intern Med 2003; 163: 585-591.

38 Nguyen HQ, Gill DP, Wolpin S, et al. Pilot study of a cell phone-based exercise persistence intervention post-rehabilitation for COPD. Int J Chron Obstruct Pulmon Dis 2009; 4: 301-313.

39 Waterhouse JC, Walters SJ, Oluboyede Y, et al. A randomised $2 \times 2$ trial of community versus hospital pulmonary rehabilitation, followed by telephone or conventional follow-up. Health Technol Assess 2010; 14: i-v, vii-xi, 1-140.

40 Antoniades NC, Rochford PD, Pretto JJ, et al. Pilot study of remote telemonitoring in COPD. Telemed J E Health 2012; 18: 634-640

41 Lewis KE, Annandale JA, Warm DL, et al. Home telemonitoring and quality of life in stable, optimised chronic obstructive pulmonary disease. J Telemed Telecare 2010; 16: 253-259.

42 Troosters T, van der Molen T, Polkey M, et al. Improving physical activity in COPD: towards a new paradigm. Respir Res 2013; 14: 115.

43 Donaire-Gonzalez D, Gimeno-Santos E, Balcells E, et al. Physical activity in COPD patients: patterns and bouts. Eur Respir J 2013; 42: 993-1002.

44 Ringbaek T, Brondum E, Martinez G, et al. Long-term effects of 1-year maintenance training on physical functioning and health status in patients with COPD: A randomized controlled study. J Cardiopulm Rehabil Prev 2010; 30: 47-52.

45 Bourbeau J, Bartlett SJ. Patient adherence in COPD. Thorax 2008; 63: 831-838.

46 Sulaiman I, Cushen B, Greene G, et al. Objective assessment of adherence to inhalers by COPD patients. Am J Respir Crit Care Med 2016; in press [https://doi.org/10.1164/rccm.201604-0733OC].

47 Papaioannou AI, Bartziokas K, Tsikrika S, et al. The impact of depressive symptoms on recovery and outcome of hospitalised COPD exacerbations. Eur Respir J 2013; 41: 815-823.

48 Papaioannou AI, Bartziokas K, Loukides S, et al. Cardiovascular comorbidities in hospitalised COPD patients: a determinant of future risk? Eur Respir J 2015; 46: 846-849. 